\title{
Radio Observations of Mira Variables, OH/IR Stars and M-Supergiants
}

\author{
Jessica M. Chapman \\ Anglo-Australian Observatory, PO Box 296, Epping, NSW 2121, \\ Australia
}

H. J. Habing

Sterrewacht Leiden, PO Box 9513, 2300 RA Leiden, the Netherlands

\author{
N. E. B. Killeen \\ Australia Telescope National Facility, PO Box 76, Epping, NSW 2121, \\ Australia
}

\begin{abstract}
We review radio maser observations of the circumstellar envelopes of oxygen-rich Mira variables, OH/IR stars and M-type supergiants.
\end{abstract}

\section{Introduction}

Towards the end of their lifetimes, most stars lose a considerable fraction of their mass through circumstellar winds. For low and intermediate mass-stars, the largest mass-loss rates of up to $10^{-4} \mathrm{M}_{\odot} \mathrm{yr}^{-1}$ occur from asymptotic giant branch (AGB) stars. These are luminous, long-period variable stars which include the Mira variables with stellar periods between 100 and 800 days, and the so-called "OH/IR" stars with stellar periods between 300 and 3000 days. Massloss rates above $10^{-5} \mathrm{M}_{\odot} \mathrm{yr}^{-1}$ are also known for a small number of massive M-type supergiant stars with stellar periods above 600 days.

AGB stars and M-type supergiants are evolved stars which have already been through several stages of nuclear burning. The stars have compact cores and extended stellar atmospheres. Strong radial pulsations in the stellar atmospheres are directly linked to the stellar mass-loss in the following way: As the star pulsates, gas in the outer atmosphere is lifted to a height of several stellar radii by pulsation-driven shock waves. At this height the temperature is sufficiently low for the condensation of solid grains. The grains are accelerated outwards by radiation pressure and momentum is transfered to the gas by grain-gas collisions. The outflowing dust and gas forms an expanding circumstellar envelope in which material is transferred from the stellar surface to the interstellar medium at a terminal velocity of typically $15 \mathrm{~km} \mathrm{~s}^{-1}$ (Goldreich \& Scoville 1976).

The AGB stars with mass-loss rates above $10^{-6} \mathrm{M}_{\odot} \mathrm{yr}^{-1}$ are generally invisible at optical wavelengths as the central stars become completely enshrouded by their circumstellar envelopes. However their envelopes can be studied at infrared and radio wavelengths. The infrared emission occurs from circumstellar 
grains which absorb and re-emit the stellar luminosity, whilst radio emission occurs from molecules which form in the circumstellar envelopes. In particular, for stars with oxygen-rich envelopes, radio maser emission may be detected from $\mathrm{SiO}, \mathrm{H}_{2} \mathrm{O}$ or $\mathrm{OH}$ molecules. Each of these lines requires different physical conditions for its excitations and so originates from a different region of the circumstellar envelope (Chapman \& Cohen 1986).

In this paper we review recent radio maser observations of the circumstellar envelopes of oxygen-rich Mira variables, $\mathrm{OH} / \mathrm{IR}$ stars and M-type supergiants. Table 1 summarizes some properties for the three groups. The parameter $R$ is the far-infrared colour defined as $\log \left(\mathrm{F}_{25} / \mathrm{F}_{12}\right)$, where $\mathrm{F}_{25}$ and $\mathrm{F}_{12}$ are the IRAS flux densities at 25 and $12 \mu \mathrm{m}$. This parameter is a useful indicator of the dust optical depth in a circumstellar envelope (van der Veen 1989).

Table 1. Properties of Miras, OH/IR Stars and M-type Supergiants

\begin{tabular}{lccc}
\hline & Miras & OH/IR Stars & M-Supergiants \\
& & & \\
\hline Period (days) & $100 \rightarrow 800$ & $300 \rightarrow 3000$ & $600 \rightarrow 1200$ \\
$\mathrm{~L}_{\text {star }}\left(\mathrm{L}_{\odot}\right)$ & $10^{3} \rightarrow 10^{4}$ & $10^{3} \rightarrow 5 \times 10^{4}$ & $>10^{5}$ \\
$\dot{\mathrm{M}}\left(\mathrm{M}_{\odot} \mathrm{yr}^{-1}\right)$ & $10^{-8} \rightarrow 10^{-6}$ & $10^{-7} \rightarrow 10^{-4}$ & $10^{-5} \rightarrow 3 \times 10^{-4}$ \\
$\mathrm{~V}_{\exp }\left(\mathrm{km} \mathrm{s}^{-1}\right)$ & $5 \rightarrow 10$ & $5 \rightarrow 30$ & $15 \rightarrow 35$ \\
$\mathrm{R}$ & $-0.4 \rightarrow 0.0$ & $-0.4 \rightarrow 0.5$ & $-0.4 \rightarrow 0.0$ \\
\hline
\end{tabular}

The Mira variables are traditionally classified as being optically visible, long-period variable stars with large amplitude pulsations $\left(\Delta \mathrm{M}_{V}>2.5\right)$. The $\mathrm{OH} / \mathrm{IR}$ stars are closely related to the Miras but extend their range of properties to thicker circumstellar envelopes, higher mass-loss rates and expansion velocities and longer stellar periods. The $\mathrm{OH} / \mathrm{IR}$ stars are detected in $18 \mathrm{~cm}$ $\mathrm{OH}$ maser emission (from one or more of the ground state transitions) and have infrared colours and luminosities which are consistent with their classification as AGB stars. ${ }^{1}$ The stars with $R>0$ are mostly not optically visible.

At present, approximately $1100 \mathrm{OH} / \mathrm{IR}$ stars are known (e.g., te Lintel Hekkert et al. 1991; Chengalur et al. 1993). A recent sensitive radio survey of the plane and bulge of our Galaxy, is expected to give a further 2000 new identifications of $\mathrm{OH} / \mathrm{IR}$ stars (Sevenster et al., these proceedings). Only a small number $(\sim 8)$ of M-type supergiant stars with masers are known. These stars are estimated to have initial masses above $10 \mathrm{M}_{\odot}$ and are characterized by their high mass-loss rates and expansion velocities but relatively thin circumstellar envelopes.

\footnotetext{
${ }^{1} \mathrm{OH}$ maser emission is also detected from a small number of hotter stars which are likely to be evolving away from the AGB. These stars are not discussed in this paper.
} 


\section{OH $1612 \mathrm{MHz}$ masers}

\subsection{The location of the $\mathrm{OH} 1612 \mathrm{MHz}$ masers}

$\mathrm{OH}$ maser emission from evolved stars is detected at a wavelength of $18 \mathrm{~cm}$ from ground state, hyperfine transitions at 1612,1665 and $1667 \mathrm{MHz}$. The masers are radiatively pumped by far-infrared emission from warm circumstellar grains. For details of the maser pumping see the review by Cohen 1989.

Aperture synthesis observations of approximately 40 sources have shown that the $\mathrm{OH} 1612 \mathrm{MHz}$ masers are located in the cool outer regions of the circumstellar envelopes, at radii of 100 stellar radii $\left(R_{\star}\right)$ or more (e.g., Booth et al. 1981; Diamond et al. 1985; Chapman \& Cohen 1986; Welty et al. 1987). At these radii, the gas temperatures are $\sim 100 \mathrm{~K}$ and the envelope kinematics are dominated by radial expansion at, or close to, terminal velocity. The strongest maser amplification occurs along the radial directions through the front and back of the circumstellar envelope and the $\mathrm{OH} 1612 \mathrm{MHz}$ spectral profiles are twin-peaked with the blue- and red-shifted peaks separated by twice the envelope expansion velocity. In most sources, the $\mathrm{OH} 1612 \mathrm{MHz}$ masers are located within a thin'spherical shell. The maser shells however generally show bright knots of emission, indicating some density variations or clumpiness in the mass outflow (Diamond et al. 1985; Welty et al. 1987).

Observationally, there is a good correlation between the $\mathrm{OH}$ shell radii and the stellar mass-loss rates (Cohen 1993). This agrees well with theoretical models in which the $\mathrm{OH}$ molecules are formed by the photodestruction of $\mathrm{H}_{2} \mathrm{O}$ by external UV photons. Stars with higher mass-loss rates and larger envelope densities provide better shielding of the $\mathrm{H}_{2} \mathrm{O}$ molecules; and so form $\mathrm{OH}$ at larger radii (Huggins \& Glassgold 1982).

\subsection{OH $1612 \mathrm{MHz}$ variability}

$\mathrm{OH} 1612 \mathrm{MHz}$ light curves have been obtained for over $200 \mathrm{Miras}$ and $\mathrm{OH} / \mathrm{IR}$ stars since Harvey et al. first showed that the $\mathrm{OH} 1612 \mathrm{MHz}$ masers are strongly saturated and radiatively coupled to the stellar pulsation cycle (Harvey et al. 1974; Schultz et al. 1978; Herman \& Habing 1985; van Langevelde et al. 1990, 1993; West et al. 1992; Chapman et al. 1993).

We have monitored the $\mathrm{OH} 1612 \mathrm{MHz}$ maser emission from a sample of 95 southern $\mathrm{OH} / \mathrm{IR}$ stars in the bulge and disk of the Galaxy, using observations taken with the Parkes 64-m radio telescope between 1988 and 1993 (Chapman et al. 1993, and in preparation). As examples, Fig. 1 shows the $\mathrm{OH} 1612 \mathrm{MHz}$ light curves for four stars in the sample. The $\mathrm{OH}$ light curves are generally asymmetric with steeper increases towards light maxima and more gradual decays towards minima. Monitoring over several light cycles has shown that the amplitudes of the light curves can vary significantly from cycle-to-cycle, (see also Gaylard \& West, these proceedings). These characteristics are also observed in the optical and infrared light curves of Mira variables providing a clear link between the two groups.

88 of the 95 stars in the Parkes sample show periodic variations with maximum/minimum flux density ratios between 1.1 and 3.8. The remaining 7 sources either have irregular light curves or show no significant variations. Of the 88 sources with measurable periods, nearly one third ( 28 per cent) have periods in 
excess of 800 days. Such long periods are not observed for optically visible Mira variables. The $\mathrm{OH} / \mathrm{IR}$ stars with periods above $\sim 800$ days appear to have well defined properties with large envelope expansion velocities $\left(15 \rightarrow 30 \mathrm{~km} \mathrm{~s}^{-1}\right)$; thick circumstellar envelopes $(R=0.0 \rightarrow 0.5)$, and large shell radii $\left(>5 \times 10^{16}\right.$ $\mathrm{cm})$. From their kinematic distances, they have an average scale height in the Galaxy of only $|z| \sim 50 \mathrm{pc}$. From these properties, we identify the longest period $\mathrm{OH} / \mathrm{IR}$ stars as AGB stars with high mass-loss rates, which belong to a young disk population with progenitor masses of at least $4 \mathrm{M}_{\odot}$.
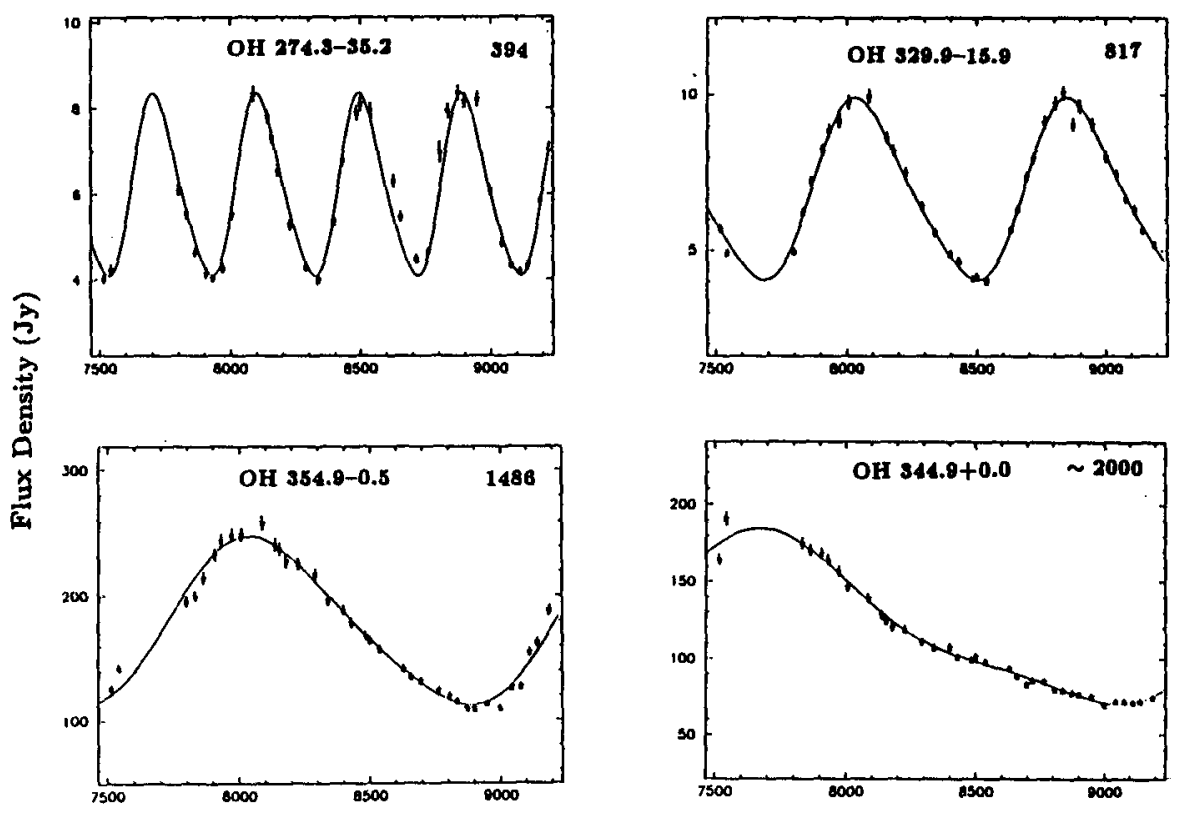

Modiffed Julian Date

Figure 1. $\mathrm{OH}$ light curves for four $\mathrm{OH} / \mathrm{IR}$ stars in the Parkes montoring programme. Best-fit stellar periods are given in the top right corners.

\section{3. $\mathrm{OH} 1612 \mathrm{MHz}$ masers and stellar distances}

The geometric distance to a Mira variable or $\mathrm{OH} / \mathrm{IR}$ star can be obtained by combining the angular and linear diameters of the circumstellar envelope. The angular diameter is obtained from aperture synthesis imaging of the $\mathrm{OH}$ maser emission, whilst the linear diameter is determined by measuring the time-delay, or "phase-lag" between the $\mathrm{OH}$ light curves from the front and back of the circumstellar envelope. For a spherically symmetric envelope, the linear $\mathrm{OH}$ shell diameter is given by the simple relation $D_{16}=\Phi / 3.86$, where $D_{16}$ is the shell diameter in units of $10^{16} \mathrm{~cm}$, and $\Phi$ is the front-back phase-lag measured in days. Typically the front-back phase lags are only a few per cent of the stellar 
period, so accurate long term monitoring is needed to determine linear diameters using this method.

The phase-lag technique was first demonstrated by Schultz et al. (1978) who obtained positive phase lags for nine sources. In a much larger programme, Herman \& Habing (1985) used the Dwingeloo 25-m radio telescope to monitor the $\mathrm{OH} 1612 \mathrm{MHz}$ emission from 60 stars over a period of five years. Angular diameters were obtained for 11 of these sources. From this subset, Herman determined the Galactic Centre distance to be $9.2 \pm 1.2 \mathrm{kpc}$. More recently van Langevelde et al. (1990) have re-analysed the Dwingeloo data These authors give phase lag diameters for 43 stars and geometric distances for 24 stars. In the best cases, the distances are accurate to $\sim 30$ per cent. Phase-lag linear diameters have also been determined by van Langevelde et al. (1993) for a few sources near the Galactic centre and by West et al. for sources in a long term monitoring programme of 14 stars at Hartebeesthoek in South Africa (1992, and in preparation).

We have used the Parkes data to determine phase-lags for 75 southern stars. The phase-lags determined using a Fourier series analysis, are between 5 and 120 days, corresponding to linear $\mathrm{OH}$ diameters between $1.3 \times 10^{16}$ and $3 \times 10^{17}$ $\mathrm{cm}$. In the best cases, the phase lag diameters are accurate to better than 10 per cent. The phase lag diameters show a clear trend for an increase of $\mathrm{OH}$ shell diameter with stellar period. This result can be understood as showing that more massive stars, with longer stellar periods, also have larger mass-loss rates and $\mathrm{OH}$ shell radii.

To determine stellar distances we (Chapman, Killeen, van Langevelde) are currently obtaining the angular diameters of the sources in the Parkes programme. As an example, Fig. 2 shows the $\mathrm{OH} 1612 \mathrm{MHz}$ spectrum and images obtained for the source $\mathrm{OH} 21.5+0.5$ from observations taken with the MERLIN array (U.K.) in November 1993. Images are shown for a number of velocity cuts across the spectrum. For each image, the velocity with respect to the local standard of rest, is given in the top left corner. The first and last images show the emission at the extreme red- and blue-shifted velocities respectively.

The images of $\mathrm{OH} 21.5+0.5$, as for other OH/IR stars, provide strong confirmation for the spherical thin-shell model of Reid et al. (1977). In this model, the $\mathrm{OH}$ masers are located in a thin spherical shell of constant radius. The strongest blue- and red-shifted emission, occurs from unresolved regions at the front and back of the circumstellar envelope. At intermediate velocities, weaker emission is detected from rings, centred on the line-of-sight through the stellar position. For well resolved sources, such as $\mathrm{OH} 21.5+0.5$, the angular diameters of the circumstellar envelopes can be accurately determined by fitting the variation in projected ring radius with line-of-sight velocity. For $\mathrm{OH} 21.5+0.5$, we obtain an angular diameter of $1.05 \pm 0.08$ arcsec.

$\mathrm{OH} 21.5+0.5$, is a good example of a high mass, long period OH/IR star. The stellar period is $\sim 1800$ days and the linear $\mathrm{OH}$ diameter, determined by us from the front-back phase-lag, is $(3.1 \pm 0.24) \times 10^{17} \mathrm{~cm}$, making it one of the largest $\mathrm{OH}$ shells known. Combining the linear and angular diameters yields a distance of $10.0 \pm 1.0 \mathrm{kpc}$ with a height above the galactic plane of only $10 \mathrm{pc}$, consistent with a young disk population. 

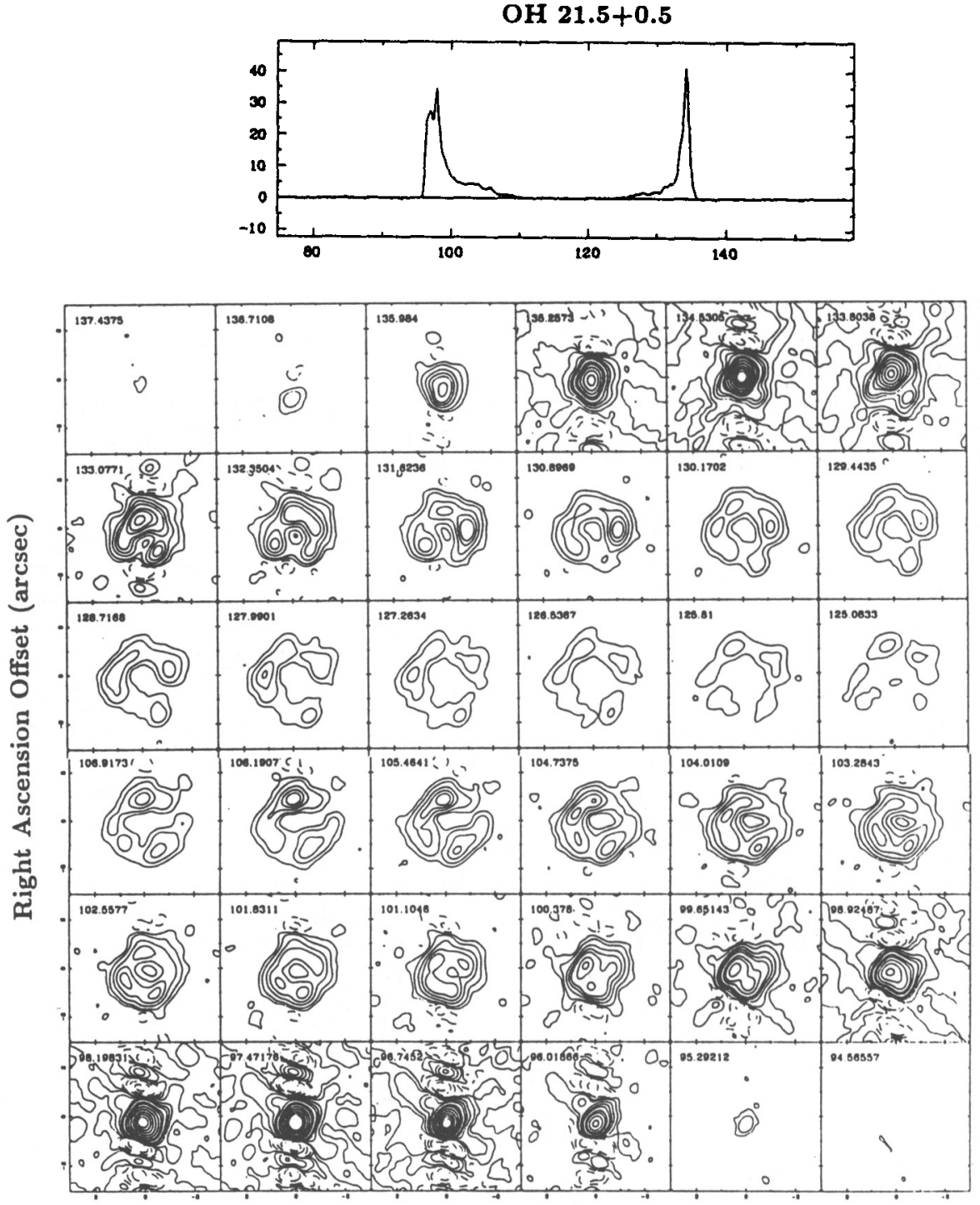

Declination Offset (arcsec)

Figure 2. $\mathrm{OH} 1612 \mathrm{MHz}$ spectrum and MERLIN images obtained in November 1993 for the source $\mathrm{OH} 21.5+0.5$ 


\section{OH mainline masers}

The $\mathrm{OH}$ mainline spectra at 1665 and $1667 \mathrm{MHz}$ are excited closer to the central stars at gas temperatures between $\sim 100$ and $400 \mathrm{~K}$. Type I OH mainline emission, with stronger maser emission at 1665 and $1667 \mathrm{MHz}$ than at $1612 \mathrm{MHz}$, is detected from Mira variables with relatively warm circumstellar dust shells. Strong mainline emission is also detected from the M-type supergiant stars.

The $\mathrm{OH}$ mainline masers of type I Mira variables and M-type supergiants have complex characteristics. Their spectral profiles are strongly circularly polarized and may show numerous spectral features, including emission at the stellar velocity. Sivagnanam (1989) has shown that the integrated $\mathrm{OH}$ mainline emission in Miras follows the stellar pulsation cycles. However, individual features may show strongly erratic variations, or flare-type activity, on timescales of months (Jewell et al. 1980; Le Squeren \& Sivagnanam 1985; Chapman et al. 1991). Such variations show that the mainline masers are partially unsaturated.

The circular polarization of the $\mathrm{OH}$ mainline masers arises from Zeeman splitting of the $\mathrm{OH}$ lines in the stellar magnetic field. Estimates of magnetic field strengths have been obtained for several sources with typical values of a few $\mathrm{mG}$ at 20-50 stellar radii (e.g., Claussen \& Fix 1982; Chapman \& Cohen 1986). For a $1 / \mathrm{r}^{2}$ dependence of the magnetic fields, the magnetic field strengths at the stellar photospheres would be around 1-10 G.

The spatial distributions of $\mathrm{OH}$ mainline masers are not straightforward to interpret. Bowers et al. (1989) found evidence for axi-symmetric distributions in several Mira variables. For the Mira variable U Orionis, Chapman et al. $(1985 ; 1991)$ found the $\mathrm{OH}$ masers to be located within clumpy structures with a typical clump size of $\sim 25 \mathrm{AU}$. The strongest mainline emission from this source occurs from a ring of radius $60 \mathrm{AU}$ which appears to be part of an expanding, tilted torus. Proper motions were measured for the $\mathrm{OH}$ masers on

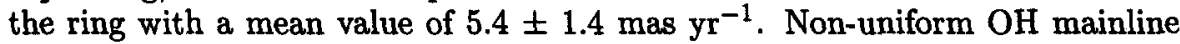
maser distributions have also been observed for the Mira variables $\mathrm{R}$ Cas and $\mathrm{U}$ Her (Chapman et al. 1994). For these sources, the strongest masers are located within partially filled thick-shells at radii between $\sim 50$ and 150 AU $(\sim 25-75$ $\mathrm{R}_{\star}$ ). The masers of $\mathrm{R}$ Cas are highly clumped and show a clear separation of blue- and red-shifted emission indicating that the circumstellar mass-loss may be axi-symmetric.

An issue which remains to be resolved is whether radial acceleration occurs to large radii in the envelopes of some sources. For $\mathrm{R}$ Cas, a comparison of the $\mathrm{OH}$ and $\mathrm{CO}$ velocity ranges indicates that strong radial acceleration may be present. The thermal $\mathrm{CO}$ emission from $\mathrm{R}$ Cas is located in the outermost parts of the envelope at $\sim 1000 R_{\star}$, and has a velocity range of $28 \mathrm{~km} \mathrm{~s} \mathbf{s}^{-1}$, almost twice that of the $\mathrm{OH}$ mainline maser emission (Bujurrabal \& Alcolea 1991; Chapman et al. 1994). An alternative hypothesis, which needs to be tested, is that the difference between the $\mathrm{CO}$ and $\mathrm{OH}$ velocity ranges are due to a strongly aspherical envelope geometry in which the different molecules trace regions of considerably different outflow velocities.

The velocity field of $\mathrm{R}$ Cas appears to be similar to that of the supergiant star VX Sgr which also has broader CO than OH maser emission. Chapman \& Cohen (1986) interpreted the $\mathrm{OH}$ mainline and $\mathrm{H}_{2} \mathrm{O}$ masers in the circumstellar envelope of VX Sgr, as being located in an accelerating thick-shell with a log- 
arithmic velocity gradient in the shell of $\epsilon \sim 0.5$. Here, we note that the $\mathrm{OH}$ mainline maser emission from VX Sgr is exceptionally variable (Chapman et al. 1995 in preparation). OH mainline spectra of VX Sgr taken in 1993 show that the maser emission to be considerably stronger and to extend over a broader velocity range than in the earlier data of Chapman \& Cohen (1986). Similar changes have also occured in the $\mathrm{H}_{2} \mathrm{O}$ spectral profiles (Bowers et al. 1992). Such changes suggest that intrinsic changes may have occurred in the velocity field of VX Sgr.

\section{4. $\mathrm{H}_{2} \mathrm{O}$ masers}

The $\mathrm{H}_{2} \mathrm{O}$ maser emission at $22 \mathrm{GHz}$ occurs from an inversion between two collisionally excited rotational states $\left(6_{16} \rightarrow 5_{23}\right)$, with radiative decay. These masers are likely to be collisionally excited at temperatures between $\sim 300$ and $1000 \mathrm{~K}$ and gas densities of $\mathrm{n}_{H 2}=10^{13}-10^{15} \mathrm{~m}^{-3}$ (Yates \& Cohen 1994). For collisional excitation, the $\mathrm{H}_{2} \mathrm{O}$ masers are expected to lie within a shell with the inner and outer shell boundaries determined by the collision rates. At the inner boundary, the maser inversion is quenched by gas thermalization at high collision rates, whilst at the outer bounday the collision rates are too low for a strong population inversion (Cooke \& Elitzur 1985; Menten \& Melnick 1991).

The $\mathrm{H}_{2} \mathrm{O}$ spectra of evolved stars mostly show two types; a double-peaked spectrum similar to the $\mathrm{OH} 1612 \mathrm{MHz}$ maser spectra or a strong single peak at the stellar velocity. The masers are likely to be unsaturated (Reid \& Menten $1990)$ and are strongly variable with erratic cycle-cycle variations. In some cases the spectral profiles have changed from double-peaked to single-peaked or vice versa.

The $\mathrm{H}_{2} \mathrm{O}$ masers are located in the inner circumstellar envelopes and probe the region where the gas is accelerated to terminal velocity. Images obtained for $\mathrm{H}_{2} \mathrm{O}$ masers in $\sim 20$ Mira variables and M-type supergiants have shown that these masers are located within 3 and $30 R_{\star}$, corresponding to $10^{14} \rightarrow 10^{15} \mathrm{~cm}$ for the Mira variables and $10^{15} \rightarrow 10^{16} \mathrm{~cm}$ for the supergiant stars (Chapman \& Cohen 1986; Lane et al. 1987; Reid \& Menten 1990; Bowers et al. 1993; Yates \& Cohen 1994; Richards et al. 1995). Observationally, the radii of the inner $\mathrm{H}_{2} \mathrm{O}$ shell boundaries increase with the stellar mass-loss rates as predicted by the collisional excitation models (Lane 1987; Yates \& Cohen 1994).

Strong evidence is seen for acceleration in the $\mathrm{H}_{2} \mathrm{O}$ shells of some sources. Fig. 3 shows the integrated $\mathrm{H}_{2} \mathrm{O}$ emission from $\mathrm{S}$ Per, taken from Richards et al. (1995). The strongest $\mathrm{H}_{2} \mathrm{O}$ emission clearly lies on a ring-like structure at a mean radius of $\sim 7 \mathrm{R}_{\star}$, with little emission from the front and back of the circumstellar envelope. The simplest explanation for such structures, is that the $\mathrm{H}_{2} \mathrm{O}$ masers are tangentially amplified, at radii where strong radial acceleration occurs. For a uniform, spherical envelope with velocity gradient $\epsilon>1$, the strongest emission occurs at the stellar velocity from a face-on ring centred on the stellar position (Chapman \& Cohen 1985). From an analysis of the individual $\mathrm{H}_{2} \mathrm{O}$ masers, Richards et al. (1995 and in preparation) model the masers as belonging to an accelerating thick-shell with inner and outer boundaries of 4 and $10 R_{\star}$, and corresponding expansion velocities of 10 and $16 \mathrm{~km} \mathrm{~s}^{-1}$. Within this shell 
the masers show striking filamentary structures, which appear to extend over considerable depths in the envelope and over several $\mathrm{km} \mathrm{s}^{-1}$.

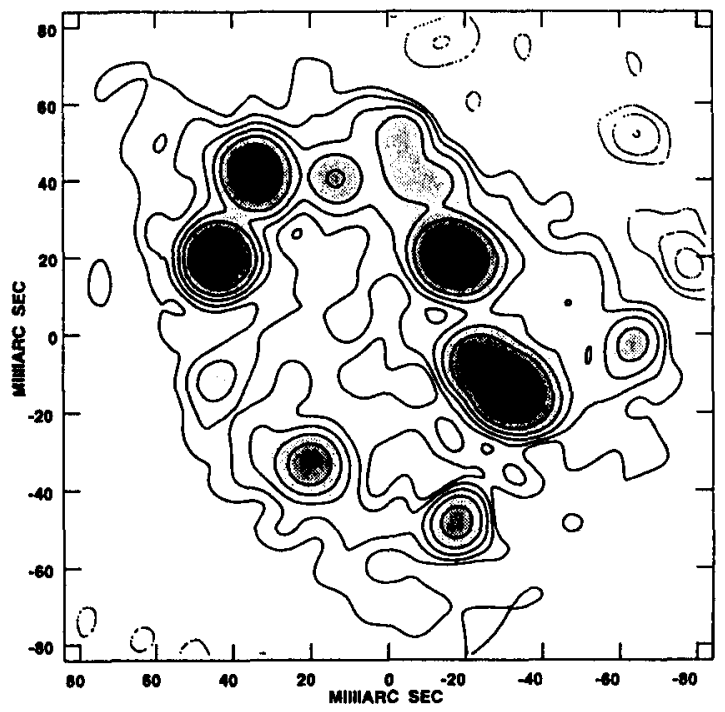

Figure 3. The integrated $\mathrm{H}_{2} \mathrm{O}$ maser emission from $\mathrm{S}$ Per, taken from Richard et al. 1995

Similar $\mathrm{H}_{2} \mathrm{O}$ ring structures are known for the semi-regular variable W Hya (Reid \& Menten 1990) and the Mira variable IK Tau (Lane et al. 1987; Yates \& Cohen 1994). In each case, the ring of $\mathrm{H}_{2} \mathrm{O}$ masers is located within $15 \mathrm{R}_{\star}$ and shows no systematic velocity gradients. The detection, in several sources, of such rings strongly supports the hypothesis that the $\mathrm{H}_{2} \mathrm{O}$ masers are located in accelerating regions rather than in aspherical or disk-like regions which should show more elliptical distributions with systematic velocity gradients.

An interesting possibility discussed by Cohen (1989) is that the most rapid changes observed in the $\mathrm{H}_{2} \mathrm{O}$ masers, which occur on timescales of weeks, may be caused by pulsation-driven shock waves, propagating through the inner envelopes at velocities of typically $30 \mathrm{~km} \mathrm{~s}^{-1}$. Such shock waves could provide an additional acceleration mechanism in the inner envelope. If this is so, then time-dependent changes related to the stellar pulsation cycle might be evident in the location and velocities of the $\mathrm{H}_{2} \mathrm{O}$ masers.

\section{SiO masers}

The $\mathrm{SiO}$ masers are located closest to the stars in the region where the onset of mass-loss occurs. The SiO molecules may act as an efficient coolant in the extended stellar atmospheres (Muchmore et al. 1987). If this is the case, then "run-away" cooling may lead to thermal instabilities giving enhanced grain for- 
mation in a two-phase medium with cooler clumps embedded within a less-dense, warmer gas.

The $\mathrm{SiO}$ maser emission is observed from several rotational transitions in excited vibrational levels, requiring temperatures above $\sim 1000 \mathrm{~K}$. The $\mathrm{SiO}$ spectra are strongly variable and linearly polarized with typical linear polarizations detected for Miras and supergiants of tens of per cent. For several sources, weak circular polarization, of a few per cent, has been also detected (Barvainis et al. 1987; McIntosh et al. 1993). The Zeeman splitting effect is very weak for $\mathrm{SiO}$ molecules (which are non-paramagnetic) and no Zeeman pairs have been identified. If the circular polarization is due to the Zeeman effect, then strong magnetic fields of tens of Gauss at the stellar photosphere are indicated. Such strong fields appear to be consistent with the weaker fields, measured at larger radii from $\mathrm{OH}$, and would have a dominant influence on the gas motions in the inner envelopes (Chapman \& Cohen 1986; Barvainis et al. 1987). However an alternative hypothesis, which needs to be further investigated, is that the circular polarization may occur in regions of much lower magnetic fields due to linearly polarized emission passing through an anisotropic medium (Nedoluha \& Watson 1993).

Early VLBI observations of SiO masers in the supergiant stars VY Cma and VX Sgr, and the Mira variable $\mathrm{R}$ Cas, showed the masers to be located within several stellar radii (Moran et al. 1979; Lane 1984; McIntosh et al. 1989). For $\mathrm{R}$ Cas, McIntosh et al. found the SiO maser emission to be located within a small number $(\sim 5)$ of clumps with each clump having well defined polarization characteristics.

Recent high angular resolution $\mathrm{SiO}$ images have been obtained for several sources. The SiO masers show a range of spot sizes from $\sim 10^{12}$ to $10^{14} \mathrm{~cm}$ (Colomer et al. 1992; Greenhill et al. 1995), and generally have no clear velocity patterns, as expected if strong turbulent motions, rather than a radial outwards expansion, dominate the kinematics close to the star. Ring-like structures centred on the stellar position have been observed for the Mira variables TX Cam and U Her (Diamond et al. 1994) and for the semi-regular variable W Hya (Miyoshi et al. 1994) again indicating strong tangential amplification. However, the acceleration of the $\mathrm{SiO}$ masers could presumably arise from both infall or outflow motions.

Greenhill et al. (1995) have studied in detail the $\mathrm{SiO}$ masers of the supergiant star VX Sgr and have obtained near-simultaneous measurements of the $\mathrm{SiO}$ maser emission and the mid-infrared dust emission. They found that near minimum light, the brightest $\mathrm{SiO}$ masers were located on a ring of radius $1.3 R_{\star}$ with an inner dust shell radius of $4.6 R_{\star}$. These observations, for the first time, clearly locate the $\mathrm{SiO}$ masers well within the inner radius of the dust shell, in the extended stellar atmosphere.

\section{Conclusions}

Radio observations of $\mathrm{SiO}, \mathrm{H}_{2} \mathrm{O}$ and $\mathrm{OH}$ masers can be used to study the detailed structures of the circumstellar envelopes of evolved stars, including the Mira variables, $\mathrm{OH} / \mathrm{IR}$ stars and $\mathrm{M}$-type supergiants. The masers are located in a sequence of layers at radii between one and 100 stellar radii, as expected from 
considerations of their excitation processes. The OH/IR stars have the most uniform circumstellar envelopes. Observations of the $\mathrm{OH} 1612 \mathrm{MHz}$ masers in these stars, show that the mass-loss is essentially spherically symmetric, with the masers located in thin-shells expanding at terminal velocity.

The circumstellar envelopes of Mira variables and M-supergiants are far less uniform. Observations of $\mathrm{SiO}, \mathrm{H}_{2} \mathrm{O}$ and $\mathrm{OH}$ mainline masers, show that the mass-loss from these stars is likely to be intrinsically clumpy or irregular. The clumpiness may be enhanced by efficient cooling processes occuring within the extended stellar atmospheres.

Several issues remain unclear, including the role of the stellar magnetic fields and of shock waves in the envelopes, and the cause of clumping and of asymmetries in Miras and supergiant stars.

Acknowledgments. Jessica Chapman thanks the scientific organizing committee for providing financial support to attend the meeting, Peter Goldreich for discussions and Jim Moran, Jim Cohen and Anita Richards for providing results prior to publication.

\section{References}

Barvainis, R., McIntosh, G. \& Read Predmore, C. 1987, Nature, 329, 613

Booth, R.S., Kus, A.J., Norris, R.P., Porter, N.D. 1981, Nature, 290, 382

Bowers, P.F., Claussen, M.J. \& Johnston, K.J. 1993, AJ 105, 284

Bowers, P.F., Johnston, K.J. \& de Vegt, C. 1989, ApJ, 340, 479

Bujarrabal, V. \& Alcolea, J. 1991, A\&A, 251, 536

Chapman, J.M. \& Cohen, R.J. 1985, MNRAS, 212, 375

Chapman, J.M. \& Cohen, R.J. 1986, MNRAS, 220, 513

Chapman, J.M., Cohen, R.J. \& Saikia, D.J. 1991, MNRAS, 249, 227

Chapman, J.M., te Lintel Hekkert, P., Killeen, N.E.B., Caswell, J.L. \& Harnett, J.I. 1993, in Lecture Notes in Physics, Astrophysical Masers, eds A.W. Clegg \& G.E. Nedoluha, Springer Verlag, 412, p345

Chapman, J.M., Sivagnanam, P., Cohen, R.J. \& Le Squeren, A.M. 1994, MNRAS, 268, 475

Chengalur, J.N., Lewis, B.M., Eder, J. \& Terzian, Y. 1993, ApJS, 89, 189

Claussen, M.J. \& Fix, J.D., 1982, ApJ, 263, 153

Cohen, R.J. 1989, Rep. Prog. Phys., 52, 881

Cohen, R.J. 1993, in Lecture Notes in Physics, Astrophysical Masers, eds A.W.

Clegg \& G.E. Nedoluha, Springer Verlag, 412, p357

Colomer, F., Graham, D.A., Krichbaum, T.P. et al. 1992, A\&A, 254, L17

Cooke, B. \& Elitzur, M., 1985, ApJ, 295, 175

Diamond, P.J., Norris, R.P., Rowland, P.R., Booth, R.S., Nyman, L.-A. 1985, MNRAS, 212, 1

Diamond, P.J., Kemball, A.J., Junor, W., Zensus, A., Benson, J. \& Dhawanm V. 1994, ApJ, 430, L61

Goldreich, P. \& Scoville, N. 1976, ApJ, 205, 144 
Greenhill, L.J., Colomer, F., Moran, J.M., Backer, D.C., Danchi, W.C. \& Bester, M. 1995, ApJ, in press

Harvey, P.J., Bechis, K.P., Wilson, W.J. \& Ball, J.A. 1974, ApJS, 27, 331

Herman, J. \& Habing, H.J. 1985, A\&AS, 59, 523

Huggins, P.J. \& Glassgold, A.E. 1982, AJ, 87, 1828

Jewell, P.R., Webber, J.C. \& Snyder, L.E. 1980, ApJ, 249,118

Lane, A.P. 1984 IAU Symp 110, p329 eds R. Fanti, K. Kellerman \& G. Setti, Reidel, Dordrecht, Holland

Lane, A.P., Johnston, K.J., Bowers, P.F., Spencer, J.H. \& Diamond, P.J. 1987, ApJ, 323, 756

Le Squeren, A.M. \& Sivagnanam, P. 1985, A\&A, 152, 85

McIntosh, G.C., Predmore, C.R., Moran, J.M., Greenhill, L.J., Rogers, A.E.E. \& Barvainis, R. 1989, ApJ, 337, 934

McIntosh G.C., Read Predmore, C. \& Patel, N.A. 1993, ApJ, 404, L71

Menten, K.M. \& Melnick, G.J. 1991, ApJ, 377, 647

Miyoshi, M., Matsumoto, K., Kameno, S., Takaba, H. \& Iwata, T. 1994, Nature, 371,395

Moran, J.M., Ball, J.A., Predmore, C.R., Lane, A.P., Huguenin, G.R., Reid, M.J. \& Hansen, S.S., ApJ, 231, L67

Muchmore, D., Nuth, J. \& Stencel, R. 1987, ApJ, 315, L141

Nedoluha, G.E. \& Watson, W.D. 1993, in Lecture Notes in Physics, Astrophysical Masers, eds A.W. Clegg \& G.E. Nedoluha, Springer Verlag, 412, p47

Reid, M.J. \& Menten, K.M., 1990, ApJ, 360, L51

Reid, M.J., Muhleman, D.O., Moran, J.M., Johnston, K.J. \& Schwarz, P.R. 1977, ApJ, 214, 60

Richards, A.M.S., Cohen, R.J. \& Yates, J.A., 1995, in Circumsteller Matter, Proceedings of ROE Centenary Symposium, Edinburgh, ed. G.D. Watt, Kluwer, Dordrecht, in press

Schultz, G.V., Sherwood, W.A. \& Winnberg, A. 1978, A\&A, 63, L5

Sivagnanam, P. 1989, Ph.D. thesis, University of Paris-Meudon

te Lintel Hekkert, P., Caswell, J.L., Habing, H.J., Haynes, R.F. \& Norris, R.P. 1991, A\&AS, 90, 327

van der Veen, W. 1989, A\&A, 210, 127

van Langevelde, H.J., van der Heiden, R. \& van Schooneveld, C. 1990, A\&A, 239,193

van Langevelde, H.J., Janssens, A.M., Goss, W.M., Habing, H.J. \& Winnberg, A. 1993, A\&AS, 101, 109

Welty, A., Fix, J.D. \& Mutel, R.L., 1987, ApJ, 318, 852

West, M.E., Gaylard, M.J., Combrinck, W.L., Cohen R.J. \& Shepherd, M.C. 1992, in Variable Stars and Galaxies, ed B. Warner, ASP Conf. Ser., 30, p277

Yates, J. \& Cohen, R.J. 1994, MNRAS, 270, 958 


\section{Discussion}

Kerschbaum: Concerning the clumps in the OH-maser emission: What is the situation regarding variability in flux and position?

Chapman: OH-maser clumps show strong, erratic variations in flux density, but are stable in position over time scales of several years at least. For one Mira variable, $\nu$ Orionis, we (Chapman, Sivagnanam \& Cohen) detected proper motions in the $\mathrm{OH}$ clumps thus showing their "ballistic" nature.

Bedding: The $\mathrm{OH}$ rings have beautiful spherical symmetry. Planetary nebulae are often asymmetric, and there is evidence that Miras themselves are asymmetric (Tuthill et al. 1994). Is it possible that the observed sphericity of $\mathrm{OH}$ masers does not trace the mass distribution, but rather reflects the symmetric radiation field from the central star?

Chapman: I think that the higher mass-loss OH/IR stars are genuinely close to being spherically symmetric, but with incompletely filled and knotty envelopes. Why post-AGB stars have bipolar morphologies is very much an open question. The answer may be related to binary systems, or to rapid evolution of the envelope structure in the post-AGB evolutionary stage.

Zijlstra: (i) The $F_{25} / F_{12}$ ratio gives a poor correlation with $\mathrm{CO}$ mass loss rates which may be due to the 12-micron flux being affected by variation in the mass loss rate. (ii) The clumpiness seen in $1612 \mathrm{MHz}$ maps is a bit deceptive: you are looking at the tangential maser which is strongly dependent on the degree of saturation of the radial maser, which in turn depends on clumps in the inner part. It seems to me that the evidence for clumpiness in the inner shell is much stronger than for clumpiness in the outer shell.

Chapman: (i) Essentially I agree! $F_{25} / F_{12}$ is only a rough indicator of mass-loss rate, but one that is easy to use given IRAS measurements. A better indicator might be the colour $\mathrm{K}[2.2 \mu \mathrm{m}]-12$. (ii) $\mathrm{OH} 1612 \mathrm{MHz}$ masers are strongly saturated. For this reason I consider the observed knots of emission at 1612 $\mathrm{MHz}$ to reflect genuine density variations.

Goldreich: (i) Do you see the amplified stellar image? (ii) What information do you have about the magnetic field in the circumstellar envelope?

Chapman: (i) VLBI observations have shown maser spots, for the extreme blueshifted emission, which do appear to amplify the stellar image. Two examples where this has been seen are $\nu$ Her (a Mira) and OH 127.8 (an OH/IR star). (ii) Magnetic fields can and have been determined from Zeeman splitting of the $\mathrm{OH}$ main lines at 1665 and $1667 \mathrm{MHz}$. Typical field strengths are $\sim 2 \mathrm{mG}$ at 50 stellar radii.

Mattei: (i) You may be interested in the 30-year light curve of VX Sgr using the AAVSO International Database, that I show in my poster. (ii) I was interested to see the short term variations you found in your radio observations of VX Sgr. Several AAVSO observers have reported variations by as much as 1.0 to 1.5 magnitudes within hours on some Mira variables. Have you found short term variability in other stars, particularly Miras? Could the visual oscillations be related to what you found in VX Sgr? 
Chapman: Firstly let me say how wonderful the AAVSO data is, allowing us to easily see long term variability trends. VX Sgr is, so far, the only evolved star known to show such rapid $\mathrm{OH}$ variations $(\mathrm{t} \sim 1 \mathrm{hr})$. I think we may find this effect in some Miras. I am fascinated to hear of corresponding optical variations in Miras. Are the radio and optical variations related? I don't know!

Busso: Is there a "canonical" explanation why some AGB stars with periods longer than $300 \mathrm{~d}$ develop OH/IR maser features and others do not? Is there any influence from the environment, or does the reason lie only in the evolution of the central star?

Chapman: Maser emission is more easily detected from stars with thicker envelopes and stronger IR flux densities. However, we (Chapman \& Habing 1995, in preparation) have found that some stars with thick envelopes have very little (but not zero) $\mathrm{OH}$. This may be related to the clumpiness of the $\mathrm{OH}$.

Feast: Can you distinguish, in individual cases, between supergiants and other $\mathrm{OH}$ sources on the basis of $\mathrm{OH}$ data alone?

Chapman: No, not on $\mathrm{OH}$ data alone. Supergiants have high expansion velocities and blue infrared colours - these two together should separate supergiants from Miras (low $V_{\text {exp }}$, blue colours) and $\mathrm{OH} / \mathrm{IR}$ stars (redder colours, higher $V_{\text {exp }}$ ). 